\title{
Book Review: The Longevity Economy: Unlocking the World's Fastest-Growing, Most Misunderstood Market
}

\author{
Magdalena Leszko* \\ Institute of Psychology, University of Szczecin, Szczecin, Poland
}

Keywords: book review, old age, economy, older adults, aging

\section{A Book Review on}

The Longevity Economy: Unlocking the World's Fastest-Growing, Most Misunderstood Market

Joseph F. Coughlin, (New York, NY: PublicAffairs), 2017, 352 pages, ISBN: 9781610396639.

We are witnessing unprecedented changes in global demography. The population of older adults is growing rapidly and this process is believed to pose considerable challenges for governments and societies worldwide. Public health, pension strains, and slower economic growth, are the most commonly cited negative effects of population aging. This gloomy scenario, however, neglects the opportunities, which do arise from the aging society, such as a growing pool of professionals willing to share their expertise and talents.

A number of books and articles have been already published with regard to the implications of the demographic shift. However, Joseph F. Coughlin's book “The Longevity Economy: Unlocking the World's Fastest-growing, Most Misunderstood Market," takes a different approach when looking at the baby boom cohort. Coughlin perceives the increasing number of older adults as an opportunity rather than a burden. The author starts his work with a very interesting, yet provoking statement-"old age is made up." What he means is that our understanding of old age is socially constructed. The definition of being old is too strictly based on biological processes and may lead to a harmful image of old age. Due to stereotypes, older adults can be perceived as incapable of work, unhealthy, sick, weak, and being afraid of technology. Unfortunately, this stereotypical view has shaped and strongly affects the society and economy.

The primary focus of the book is older adult population as a growing sector of customers. Coughlin, as the founder and director of the MIT AgeLab, owns substantial experience in aging and technology. The AgeLab is a multidisciplinary research program created to gain more knowledge about the role of technology in older adults' lives and how it can improve their everyday functioning. Throughout the book Coughlin juxtaposes interesting examples of companies that embraced or failed the aging demographics. For example, certain drugstores reorganized products in a way that they can be easily accessible for older adults with vision or mobility impairments. Other businesses failed by introducing products with a confusing operating system. Coughlin emphasizes that many firms are not prepared for such a demographic transition, do not realize that older adults are dominant spenders, and criticizes them for not responding to the growing needs of the aging population.

"The Longevity Economy" is broken into two parts and 8 chapters. The first part of the book addresses a new way of thinking of old age. In the introduction, the author provides the reader 
with an overview of the current situation of older adults in the United States and explains why the population of older adults is growing. In the first chapter Coughlin describes how old age was historically formed and how the notion of being old changed throughout the nineteenth and twentieth centuries. This chapter also summarizes the origin of Social Security, its rules as well as controversies surrounding the program. The second chapter discusses the most common, widely accepted myths regarding the aging population. In addition, Coughlin presents examples of businesses' failures to meet older adults' needs. The next chapter is dedicated to women, as not only women comprise a significant majority of older adults, but also because they control the majority of household spending (among all age groups, women influence $64 \%$ of consumer purchases). The first part ends with a discussion of "The Villages" and other age segregated communities. The author elaborates on their origins and factors influencing older adults' lifestyles.

The second part of the book focuses on coping with misconceptions of old age and creating products that will empower older adults. The author draws upon his experience and outlines solutions that could strengthen the power of the senior market. In each chapter he presents vivid examples of what strategies work and what could be improved. Coughlin argues that it is important to stop treating older adults as patients and start looking at them as customers. Unfortunately, the idea that older adults are weak and have no aspirations is reinforced in the culture and in turn, is very costly to businesses which produce mainly health-related products. The author goes on to share insights on how to use older adults' potential and encourage them to do meaningful activities.

Coughlin concludes that many firms should study the longevity market to test their assumptions about older adults' behaviors. The needs of older adults are yet to be discovered, as the older adults often times don't know what they need until the product is offered to them. Just like other consumer products, these should also be appealing, exciting and easy to use. Equally important is to realize that older adults are not afraid of technology. The author also advises not to trust your intuition but rather study lead users, which will allow the companies to gain a better understanding of how the products can be received by wider target group.

One aspect that I wish was scrutinized more in this book is a more in-depth discussion on how the usage of technology could encourage older adults to take preventative steps to enjoy independent lives longer.

Coughlin's main message lies in the importance of overcoming the myths related to aging. It is important to recognize that consumer demands are shifting and older adults are a diverse group of consumers. With an increasing number of older adults, health-and-safety products are needed, but older adults also want to purchase things and use services that improve their quality of life. This book captivates one's attention with thoughtful message supported by real world examples. Anyone interested in aging and needs of the aging population would find a great value in this book.

\section{AUTHOR CONTRIBUTIONS}

The author confirms being the sole contributor of this work and has approved it for publication.

Conflict of Interest Statement: The author declares that the research was conducted in the absence of any commercial or financial relationships that could be construed as a potential conflict of interest.

Copyright (c) 2018 Leszko. This is an open-access article distributed under the terms of the Creative Commons Attribution License (CC BY). The use, distribution or reproduction in other forums is permitted, provided the original author(s) and the copyright owner(s) are credited and that the original publication in this journal is cited, in accordance with accepted academic practice. No use, distribution or reproduction is permitted which does not comply with these terms. 